\title{
Erarbeitung von Lösungsansätze von Zielkonflikten bei Pacht - und Bewirtschaftungsverträgen in der deutschen Weinwirtschaft
}

\author{
Michael Rohrer und Jon H. Hanf \\ Hochschule Geisenheim University, 65366 Geisenheim, Germany
}

\begin{abstract}
Durch die allgemeine Zunahme von Kurzurlaubsreisen sieht auch die Weinbranche in Europa immer mehr die wirtschaftlichen Vorteile und die damit einhergehenden positiven Synergien zwischen Wein und Tourismus. Das in unseren Breiten noch sehr junge Thema Weintourismus beschränkt sich in wissenschaftlichen Arbeiten meist auf die Angebotsstruktur und nicht auf die Erwartung der Konsumenten. In dieser Untersuchung wurden die Erwartungen von Weintouristen analysiert, um die aus der Literatur und die durch Experteninterviews gewonnenen Erkenntnisse für Weinbaugemeinden als Empfehlung zu Grunde legen zu können. Es zeigte sich, dass es nur eine geringe Zahl von Weinreisenden gibt, die explizit des Weines Willen in Durch den Abschluss eines Pacht- und Bewirtschaftungsvertrages entsteht eine vertikale Kooperation zwischen dem Weingut und dem Traubenproduzenten. Aus Sicht der Genossenschaft als Unternehmen ist diese vertikale Kooperation ebenfalls durch den Liefervertrag für Premiumweine zu erkennen. In der Beziehung zwischen beiden Vertragspartnern entstehen mitunter auch Probleme bzw. Konflikte. Diese können aus den unterschiedlichen persönlichen Zielen oder Interessen der Vertragspartner resultieren. Weitere Möglichkeiten für die Herkunft von Problemen können die unterschiedlichen Ansichten über den qualitativen Weinanbau oder mangelende Umsetzung der vertraglich festgelegten Vorgaben sein. Das Ziel war es, diese Konflikte und Probleme durch Interviews mit Entscheidungsträgern der Weinwirtschaft zu erkennen und zu analysieren. Die Ergebnisse zeigen, dass die meisten Probleme auf die Transaktionskostentheorie und auf die Prinzipal Agent Theorie zurückzuführen sind.

Contract farming is gaining in importance in the German wine business. However, this in not limited to grape producers and wineries but also cooperatives engage increasingly in contract production. Particularly for the production of premium wines cooperatives offer their members production contracts. However, within these relationships problems and conflicts evolve. Sources could be different aims or intensions of both sides. Further problems might result from different ideas about the production of wine quality and / or regarding the monitoring of quality measurements. Aim of this paper is to explore and analyze problems and conflicts which result from production contracts. In-depth interviews have been conducted. Their results show that the majority of problems are resulting from transactions costs and problems related to the principal agent setting.

L'agriculture contractuelle gagne en importance dans le monde du vin allemand. Cependant, ceci ne se limite pas aux producteurs et les caves raisins mais de façon coopératives se engagent de plus en plus dans la production du contrat. En particulier pour la production de vins haut de gamme offrent coopératives membres Leurs contrats de production. Cependant, les relations problèmes et conflits au sein de la synthèse évoluer. Sources pourraient être différents objectifs et intentions des deux côtés. D'autres problèmes peuvent résulter de différentes idées sur la production de la qualité du vin et / ou qui concerne le suivi des mesures de la qualité. Objectif de cet article est d'explorer et d'analyser les problèmes et les conflits qui résultent de contrats de production. Des entrevues approfondies ont été menées. Leurs résultats montrent fait la majorité des problèmes sont issus de coûts et les problèmes liés à la mise de principal agent transactions.
\end{abstract}

\section{Einleitung}

Im Bereich des Agrarsektors gibt es viele vertikale Beziehungen sowie vertikale Integration von einzelnen Produktionsstufen. In einigen Unternehmen der Weinwirtschaft ist der Wandel hin zur Form der vertikalen Kooperation ebenfalls zu erkennen. Ein Teil der Traubenproduktion erfolgt in den Unternehmen durch einzelne, meist kleinere Winzerbetriebe. Die Gründe für die Kooperation bei der Traubenproduktion können beispielsweise Zeit- und Produktionskostenvorteile, höhere Effizienz und Risikoverteilung sein. Viele Weingüter schließen daher sogenannte Pacht- und Bewirtschaftungsverträge mit den
Winzern ab, um sich die Anlieferung der Trauben zu sichern. In Winzergenossenschaften kann es ebenso zu zusätzlichen Verträgen mit den Mitgliedern kommen. Diese Lieferverträge werden bei der Produktion von Premiumweinen oder Selektionsweinen mit einzelnen Mitgliedern abgeschlossen. In den Verträgen können ebenfalls Vorgaben an die Bewirtschaftung der Rebfläche getätigt werden.

Die Grundstrukturen und die rechtlichen Inhalte der Pacht- und Bewirtschaftungsverträge sind im deutschen Weingesetz weitestgehend festgelegt. Neben den vertraglichen Inhalten gibt es weitere Möglichkeiten, die Qualität der Trauben und das Verhalten der Akteure in 
der Kooperation zu beeinflussen. Dies kann beispielsweise über monetäre Anreize oder weichen Faktoren (z. B. Lob oder Anerkennung) sowie durch Vertrauen, die persönliche Beziehung, die Nutzung von Macht oder informelle Verträge erfolgen.

In der Beziehung zwischen beiden Vertragspartnern entstehen mitunter auch Probleme bzw. Konflikte. Diese können aus den unterschiedlichen persönlichen Zielen oder Interessen der Vertragspartner resultieren. Weitere Möglichkeiten für die Herkunft von Problemen können die unterschiedlichen Ansichten über den qualitativen Weinanbau oder mangelende Umsetzung der vertraglich festgelegten Vorgaben sein. In der Beziehung zwischen dem Winzer und dem Unternehmen liegen zudem Informationsasymmetrien vor. Dies verursacht Kosten ("Agency-Kosten"), um die Informationsasymmetrien zwischen den Vertragspartnern zu beseitigen. Die AgencyKosten beziehen sich auf die Kosten der Kontrolle und Überwachung des Vertragspartners sowie auf die Schaffung von Anreizen. Mit Hilfe der Neuen Institutionenökonomie können weitere Zielkonflikte und Probleme in einer Vertragsbeziehung erklärt werden.

Ziel dieser Arbeit ist es, durch Interviews bzw. Befragungen von Entscheidungsträgern in Unternehmen der Weinwirtschaft (Weingüter, Weinkellereien und Winzergenossenschaften), bestehende Möglichkeiten zur Qualitätsbeeinflussung in einer Kooperationsbeziehung zu ermitteln. Desweiteren sollen die hierbei entstehenden Zielkonflikte und Probleme identifiziert werden. Mit Hilfe der theoretischen Ansätze soll versucht werden, deren Herkunft oder Ursache zu erklären. Im Anschluss sollen Empfehlungen und strategische Lösungsansätze gegeben werden, um Möglichkeiten zur Erzielung der gewünschten Traubenqualität in einem Pacht- und Bewirtschaftungsoder Liefervertrag aufzuzeigen. Im Rahmen dieser Arbeit wurden daher insgesamt 23 Weingüter inklusive einer Weinkellerei sowie Winzergenossenschaften befragt.

\section{Ausgestaltungen von vertikalen Kooperationen}

\subsection{Kooperation und verträge}

In der Agrar- und Ernährungsbranche gibt es viele verschiedene Ausprägungsformen von Kooperation. Der Begriff "Kooperation" und die differenzierten Formen werden in der Literatur auf unterschiedlichste Weise definiert und beschrieben, wobei sich viele Inhalte der Definitionen und Begrifflichkeiten überschneiden (Engels A., 2002). Allgemein kann man unter dem Begriff Kooperation eine Form der freiwilligen, zwischenbetrieblichen Zusammenarbeit von mindestens zwei Unternehmen unter Wahrung ihrer wirtschaftlichen und rechtlichen Selbstständigkeit verstehen (Hanf / Dautzenberg, 2008). Solche Organisationsformen werden auch hybride Organisationsformen oder auch partielle Integration genannt (Picot et al., 2001).

Lieferverträge, Produktionsverträge und/oder vertragliche Bindungen zwischen Landwirten und Abnehmern sind typische hybride Organisationsformen. In der Landwirtschaft haben sich unterschiedliche Ausprägungsformen von produktionsstufenübergreifenden Verträgen entwickelt, von einfachen Vermarktungsverträgen ("marketing contracts") bis hin zu engen Arbeitsverhältnissen mit hoher Bindungsintensität ("production contracts") (Spiller / Schulze, 2006). Bei einem "marketing contract" handelt es sich um eine zeitlich terminierte Vereinbarung vor Produktionsbeginn eines Gutes. Der Produzent verpflichtet sich, eine im Vertrag festgelegte Menge oder Qualität eines Produktes dem Vertragspartner zu liefern oder zu verkaufen und erhält dafür den zuvor vertraglich festgelegten Preis. Die Entscheidungsrechte sowie das Risiko liegen bis zur Lieferung beim Produzenten. Bei einem "production contract" werden die Bereitstellung von Vorleistungen (z. B. Maschinen, Saatgut, etc.), die Vorgaben an die Bewirtschaftung, die Qualität und Menge eines Gutes sowie die Vergütung bzw. den Ausgleich für den Produzenten vertraglich festgelegt. Der Vorteil liegt darin, dass der Produzent eine garantierte Abnahme des erzeugten Gutes und ein stabiles Einkommen hat sowie die Fachkenntnisse und Beratung des Abnehmers nutzen kann (Kunkel et al., 2009a; 2009b).

In der Weinwirtschaft werden solche Produktionsverträge aus rechtlicher Sicht als Pacht- und Bewirtschaftungsverträge bezeichnet. Bei einem Pacht- und Bewirtschaftungsvertrag ist der Pächter, z. B. ein Weingut, Eigentümer der Rebfläche. (Steinmetz, 2009) Der Pächter trägt alleine das unternehmerische Risiko (witterungsbedingte Ertragsausfälle, Schädlingsbefall, etc.). Dieser entscheidet über alle weinbaulichen Verfahren, trägt die Verantwortung für die Traubenproduktion und das Lesegut gehört ihm zu 100 Prozent. Diese Gegebenheiten müssen aus den vertraglichen Inhalten hervorgehen, damit das Weingut als "Erzeuger" der Trauben gilt. (Oberhofer, 2007) Die Bezeichnung des Unternehmens als "Weingut" und die Vermarktung der Weine unter der Angabe "Gutsabfüllung" oder "Erzeugerabfüllung" auf dem Etikett sind nur dann zulässig, wenn das Weingut der Erzeuger ist, und das unternehmerische Risiko sowie die Verantwortung der Urproduktion auf Seiten des Pächters (Weingut) liegen. Eine weitere Form ist der reine Bewirtschaftungsvertrag. Hier trägt der Bewirtschafter das alleinige Risiko und er ist für die Urproduktion verantwortlich. Generell ist dieser frei in allen Entscheidungen mit Blick auf die Bewirtschaftung der Rebfläche und er wird ausschließlich nach Qualität und Menge der Trauben entlohnt. Die Bezeichnung des Unternehmens als "Weingut" ist hier nicht mehr zulässig, da das unternehmerische Risiko beim Bewirtschafter liegt und die Bezahlung nach Menge und Qualität erfolgt. (Steinmetz, 2009).

In Winzergenossenschaften können ebenfalls spezielle Vereinbarungen bzw. Verträge für die Bewirtschaftung der Rebfläche mit Mitgliedern getätigt werden. Zu beachten ist im Rahmen dieser Arbeit, dass es sich bei Winzergenossenschaften aufgrund der rechtlichen Bestimmungen eines Pacht- und Bewirtschaftungsvertrages nicht um eine solche Art von Vertrag handelt. Es werden individuell gestaltete Produktions- bzw. Lieferverträge für bestimmte Selektions- oder Premiumweine mit den Mitgliedern abgeschlossen. Vorgaben an die Bewirtschaftung, das Pflanzgut, Lesedatum, Menge und Qualität 
sowie die Vergütung sind vertraglich geregelt. Die Inhalte werden meist von der Geschäftsleitung der Genossenschaft vorgegeben.

\subsection{Kooperationen im Licht der Neuen Institutionenökonomik}

Da die Akteure unterschiedlichen Ziele und persönlichen Interesse, welche oft nicht mit den Zielen und Interessen des jeweiligen Transaktionspartners übereinstimmen, verfolgen, resultieren Probleme und Konflikte bei einer vertraglichen Austauschbeziehung über Pacht- und Bewirtschaftungsverträge zwischen dem Traubenproduzenten und dem Weingut oder der Winzergenossenschaft. Da diese in der empirischen Studie mit Hilfe der Transaktionskostentheorie, der Theorie der Verfügungsrechte sowie der Prinzipal-Agent-Theorie dargestellt und analysiert werden, soll die Kernaussagen dieser theoretischen Ansätze in den folgenden Absätzen erarbeitet werden. Die Theorie der Verfügungsrechte (Property Rights) befasst sich mit der Zusammensetzung bzw. Konsistenz von Eigentumsrechten, die jedes Individuum einer Wirtschaft besitzt (Demsetz, 1967). Der Wert bei der Übertragung der Verfügungsrechte ist davon abhängig, welche Bereiche der Rechtebündel der Verfügungsrechte auch tatsächlich übergeben werden, um diese nutzen zu können (Furubotn / Pejovich, 1972). In der Regel ist es unmöglich, vollständige Verträge zu verfassen, da bestimmte Umstände und Ungewissheiten nicht vorhersehbar sind oder es einfach $\mathrm{zu}$ teuer ist, sie alle ausreichend im Detail zu spezifizieren (Hendrikse / Bijman, 2002). Wenn die Verfügungsrechte nun aufgrund unvollkommener Verträge nicht klar verteilt sind, werden beide an der Transaktion beteiligten Akteure versuchen, sich die nicht-spezifizierten Rechte anzueignen und zu ihrem Vorteil zu nutzen. Es kann ex-post zu teuren Nachverhandlungen kommen. Besonders problematisch ist, wenn es sich ex-ante um eine transaktionsspezifische Investition handelt und aufgrund opportunistischen Verhaltens die Gefahr des "Hold-up" besteht. Wer sich ex-post in welcher Verhandlungsposition befindet und dadurch von seinem Recht Gebrauch machen sowie deren Macht einsetzen kann, wird durch die Verteilung der Verfügungsrechte bestimmt.

(Hendrikse / Bijman, 2002; Hart / Moore, 1990) Die Transaktionskostentheorie hat ihren Ursprung aus ökonomischen Beiträgen der 30er Jahre von Coase (1937). Die Annahme von Coase war, dass die Nutzung eines Koordinationsmechanismus wie Markt oder Hierarchie nicht kostenlos sei, sondern bestimmte Kosten verursachen, nämlich sogenannte Transaktionskosten (Engels A., 2002). In Anlehnung an Williamson (1990) lassen sich Transaktionen in Faktorspezifität (Spezifität), Unsicherheiten, der sie ausgesetzt sind und Häufigkeit unterteilen. Dies führt dazu, dass es in der Regel nicht möglich ist, vollständige Verträge zu verfassen (Hendrikse / Bijman, 2002). Neben diesen sowie dem Kostenproblem innerhalb der Austauschbeziehung ist ein weiteres Problem ist das Vorliegen von Informationsasymmetrien.

Die Prinzipal-Agent-Theorie befasst sich mit der Analyse und Gestaltung der arbeitsteiligen Beziehung zwischen Auftraggeber (Prinzipal) und Auftragnehmer (Agent). Im Mittelpunkt der Prinzipal-Agent-Theorie stehen potenzielle Zielkonflikte, welche aus dem
Vertragsverhältnis bzw. der Kooperationen zwischen zwei oder mehreren Personen resultieren (Schramm et al., 2004). Sie erklärt bestimmte Beziehungsprobleme ("Agency-Probleme") aus Interessenskonflikten, welche aufgrund der asymmetrischen Informationsverteilung zugunsten des Agenten entstehen (Schreyögg, 2003). Der Prinzipal hat ein Informationsdefizit gegenüber dem Agenten. Die Beziehung zwischen Prinzipal und Agent ist dadurch gekennzeichnet, dass der Prinzipal dem Agenten zur Erfüllung seiner Aufgabe Entscheidungsund Handlungskompetenzen überträgt (Jensen / Meckling, 1976). Um den Problemen der asymmetrischen Informationsverteilung entgegen $\mathrm{zu}$ wirken ist der Prinzipal darauf angewiesen, vertragliche Vereinbarungen oder Organisationsregeln zu erstellen und damit das Verhalten des Agenten so zu disziplinieren, dass es auch der Interessenlage des Prinzipals entspricht (Picot / Dietl, 1993). Desweiteren kann der Prinzipal durch die Einführung geeigneter Kontroll- und Koordinationsinstrumente sowie Anreizmechanismen den Agenten motivieren, in seinem Sinne zu handeln (Jensen / Meckling, 1976). Anreizmechanismen, die das Verhalten des Agenten innerhalb der Vertragsbeziehung beeinflussen sollen, können in Form von positiven Anreizen (Belohnungen) oder negativen Anreizen (Sanktionen) sein (Schreyögg, 2003).

Betrachtet man die meisten Definitionen von Macht, die Macht erklären als die Fähigkeit eines Unternehmens, die Entscheidungsvariablen in der Strategie eines anderen Unternehmens kontrollieren zu können (El-Aasary / Stern, 1972), so sieht man die enge Verbindung zu den oben angesprochenen Anreizmechanismen. Die Sozialpsychologen French und Raven (1959) haben Macht in fünf Kategorien eingeteilt, welche die verschiedenen Machtgrundlagen oder ihre Herkunft einordnen sollen. Diese sind die rechtliche Macht ("legitime power"), Macht durch Vorbildcharakter ("referent power"), Macht durch Wissen ("expert power"), Macht durch Zwang ("coercive power") und Macht durch Belohnung ("reward power"). Eine weitere in der Literatur diskutierte ist die Macht durch Informationsvorsprung ("informational power") (Raven / Kruglanski, 1970).

Desweiteren wird im Kontext von Kooperationen und ihren Beziehungsstrukturen der Aufbau von Vertrauen befürwortet, da es die Komplexität und die damit verbundenen Unsicherheiten mindern kann (Picot et al., 2001). Das Problem von Vertrauen besteht darin, dass die Gefahren einer vertrauensvollen Vorleistung und einer hieraus resultierenden Verwundbarkeit nicht ausgeräumt sind und derjenige, der vertraut, nicht ausschließen kann, dass sich der Transaktionspartner nicht an die vereinbarten Vorgaben halten wird. Die Erbringung von Vorleistungen kann in materieller und/oder immaterieller Form erfolgen wie z. B. Investitionen, Know-HowTransfer, Einräumen von Exklusivrechten, Bereitstellung von Personal (Kühlmann, 2008).

\section{Empirische studie in der weinwirtschaft}

\subsection{Stichprobe und methodik}

Im Rahmen dieser Studie wurden daher insgesamt elf Weingüter inklusive einer Weinkellerei aus den 
Anbauregionen Baden, Pfalz, Rheinhessen und Rheingau befragt. Diese kooperieren mit Winzern in Form von Pacht- und Bewirtschaftungsverträgen. Desweiteren wurden für die Untersuchung zwölf Winzergenossenschaften aus dem Anbaugebiet Baden befragt. Die Befragung wurde mit einem Entscheidungsträger (Geschäftsführer, Eigentümer, Qualitätsmanager oder Produktionsleiter) des jeweiligen Unternehmens durchgeführt und als Befragungsform diente dabei das Interview.

Die Einzelinterviews wurden mit einem Interviewleitfaden durchgeführt, dessen Befragungstechnik aus der qualitativen empirischen Sozialforschung stammt (Kromrey, 2000). Bei der Erstellung des Leitfadens wurden für die Untersuchung fünf relevante Themenbereiche aus der Theorie abgeleitet und Fragen zu den einzelnen Themenblöcken formuliert. Bei der Befragung handelt es sich um eine offene, teilstandardisierte Befragung, d. h. es werden keine Antwortmöglichkeiten vorgegeben und der Interviewte kann frei antworten, berichten oder erklären. (Mayring, 2002) Zu Beginn des Gesprächs wurde eine kurze Einleitung zum Thema und Ziel der Untersuchung. Die aufgezeichneten Gespräche wurden zur Auswertung zunächst transkribiert. Jedes Interview wurde mit Großbuchstaben durchnummeriert. Die Aussagen der einzelnen Interviewpartner wurden den entsprechenden Themenblöcken des Interviewleitfadens zugeordnet. Im nächsten Schritt wurden gleiche bzw. sinngemäße Aussagen zu einer allgemeinen, vereinheitlichten Kernaussage zusammengefasst. Im Anschluss werden die Kernaussagen durch einige Beispielaussagen und Zitate der Interviewpartner untermauert und verdeutlicht. (Mayring, 2002).

\subsection{Ergebnisse und diskussion}

\subsubsection{Gründe für Pacht- und Bewirtschaftungsverträge}

Die Ergebnisse der Weingüter lassen erkennen, dass Kooperationen mit Traubenproduzenten über Pacht- und Bewirtschaftungsverträge hauptsächlich aufgrund von ökonomischen Zielen erfolgen. Die Weingüter versuchen Kostenvorteile durch Kooperation mit den Winzern auszunutzen. Diese entstehen aus den Skaleneffekten durch die bessere Auslastung der Kapazitäten (z. B. Lagertanks, Abfüllanlage). Desweiteren werden ökonomische Vorteile durch Spezialisierung bzw. Arbeitsteilung erzielt. Der Winzer hat durch die Auslastung seiner Kapazitäten häufig die Möglichkeit, die Traubenqualität für Basisweine günstiger $\mathrm{zu}$ produzieren. Viele Weingüter konzentrieren sich deshalb auf die Produktion von qualitativ höherwertigen Trauben. Dies ist allerdings mit einem höheren Arbeitsaufwand vor allem im Weinberg verbunden. Ein Großteil der befragten Weingüter hat angegeben, dass die Kapazitätsgrenzen bei der Traubenproduktion erreicht sind. Das Wachstum des Unternehmens ist jedoch meistens nur über eine Erhöhung der Betriebsrebfläche möglich, da hohe Preissteigerungen nicht einfach durchzusetzen sind bzw. zu Umsatzeinbrüchen führen können. Um eine Betriebsvergrößerung mithilfe einer Ausweitung der Betriebsfläche $\mathrm{zu}$ realisieren, müssen hohe Investitionen in Maschinen und Arbeitskräfte getätigt werden. Dies würde folglich zu einer Erhöhung der Fixkosten führen. Aufgrund der Flächenproblematik ist es in einigen Anbaugebieten schwierig, eine akzeptable Fixkostendegression zu erreichen. D. h., die Fixkosten pro Stück könnten nicht über eine Ausweitung der Fläche und die hierdurch größere Weinmenge gesenkt werden. Deshalb ist es aus ökonomischer Sicht sinnvoll, Trauben über Pachtund Bewirtschaftungsverträge $\mathrm{zu}$ beziehen, anstatt der eigenen Bewirtschaftung der Rebfläche. Außerökonomische Ziele, wie der Erhalt des Status "Weingut" und dem hiermit verbundenen Image, werden lediglich von zwei Interviewpartnern als weiteren Grund für Pacht- und Bewirtschaftungsverträge genannt. Diese spielen eine eher untergeordnete Rolle bei der Entscheidung bezüglich des Abschlusses eines Pacht- und Bewirtschaftungsvertrages.

Genossenschaften verfolgen mit der Produktion einer Premiumlinie ökonomische und außerökonomische Ziele. In den meisten Fällen wird das Ziel verfolgt, einen höheren Gewinn zu erwirtschaften, was auch zur Existenzsicherung der Genossenschaft beiträgt. Mit höheren Weinqualitäten kann in der Regel auch ein höherer Preis am Markt erzielt werden. Dieser Absatzmarkt (meist Gastronomie \& Fachhandel) ist jedoch nicht so groß wie der von Basisweinqualitäten, die in der Regel in größeren Mengen im LEH abgesetzt werden können. Aus diesem Grund liegt der Anteil der Premiumflächen unter zehn Prozent der gesamten Rebfläche der Genossenschaften. Die außerökonomischen Ziele sind bei den Genossenschaften im Vergleich zu den Weingütern von größerer Bedeutung. Alle befragten Interviewpartner der Genossenschaften begründen die Produktion einer Premiumlinie mit der verbesserten Reputation und des Images des Unternehmens.

\subsubsection{Probleme und Konflikte bei Pacht- und Bewirtschaftungsverträge}

Die Ergebnisse der Genossenschaften machen deutlich, dass alle über eine in der Satzung verankerte Erzeugerund Qualitätsrichtlinie verfügen. Aus Sicht der Theorie der Verfügungsrechte wird das Recht zur Nutzung der Ressource beeinflusst. Der Vorstand bzw. die Geschäftsführung werden hierdurch in ihrer Position gestärkt, und ihr Einfluss bei den Tätigkeiten der Genossenschaft erhöht. Sie erreichen durch die "Richtlinie" mehr rechtliche Macht bzw. Positionsmacht, um Sanktionen gegen ein Mitglied auszusprechen. Falls ein Mitglied nicht dazu bereit ist, die Vorgaben dieser Erzeuger- und Qualitätsrichtlinie zu akzeptieren, kann die Person von der Genossenschaft ausgeschlossen werden. Ein angehendes Mitglied würde in diesem Fall nicht in die Genossenschaft aufgenommen werden. Durch die Richtlinie werden die Rechte des einzelnen Mitgliedes in gewisser Weise eingeschränkt. Dies ermöglicht es der Geschäftsführung, die Ziele und Interessen der Genossenschaft als Unternehmen zu verfolgen. Zudem dient die Richtlinie auch dazu, einen gewissen Qualitätsstandard innerhalb der Genossenschaft $\mathrm{zu}$ erreichen und die Genossenschaft als Unternehmen gegenüber der Konkurrenz wettbewerbsfähig zu machen. Jedoch ist nicht auszuschließen, dass die Vorgaben nicht immer im Sinne aller Mitglieder sind.

Die Aussage von Interviewpartner T (13:10 min) aus den Ergebnissen der Genossenschaften lässt ein Problem 
aus der Prinzipal-Agent-Theorie erkennen. Durch die Produktion von Bioweinen kann eine Nische bedient werden, in der eine gute Wertschöpfung für die Genossenschaft $\mathrm{zu}$ erzielen ist. Die Mitglieder sind zunächst nicht bereit zu investieren und ihren Betrieb auf biologischen Anbau umzustellen. Die Umstellung von konventionellem Anbau auf biologischem Anbau stellt eine langfristige Investition für den Winzer dar. Zwischen der Genossenschaft und den Winzern liegen außerdem Informationsasymmetrien in Form von "Hidden Intention" vor. Es besteht die Gefahr des "Hold-up". Die Mitglieder sind erst durch Anreizmechanismen, wie z. B. Garantieversprechen (Abnahme der Trauben über bestimmte Jahre) und deutlich höhere Auszahlungspreise zu motivieren, biologischen Anbau zu betreiben. Durch diese langfristigen Garantieversprechen wird die Gefahr des "Hold-up" aus Sicht der Mitglieder verringert, und es kommt zu einer Investition bzw. Umstellung auf biologischen Anbau. In diesem Zusammenhang spielen der von einigen Interviewpartnern angesprochene Generationswechsel und die Denkweise der Mitglieder ebenfalls eine Rolle.

Die Ergebnisse der Genossenschaften und Weingüter machen deutlich, dass eine Selektion der Mitglieder bzw. der Vertragspartner für den Abnehmer der Trauben sehr wichtig ist. Beachtet werden muss, dass vor Vertragsabschluss Transaktionskosten für die Suche der geeigneten Vertragspartner und die Informationsbeschaffung entstehen. Anzunehmen ist, dass die Kosten auf Seiten der Genossenschaften geringer sind, als bei den Weingütern, da nur Mitglieder der Genossenschaft für eine vertraglich geregelte Traubenproduktion in Frage kommen. Die Geschäftsführung kann bei der Auswahl der Mitglieder Erfahrungswerte vorheriger Jahre sowie Informationen in Bezug auf den Winzer nutzen. Die Verantwortlichen der Genossenschaft kennen ihre Winzer in der Regel sehr gut und wissen, welche Winzer die Voraussetzung haben, um die Vorgaben der Bewirtschaftung zu erfüllen bzw. umzusetzen. Außerdem sind sie in der Lage, besser beurteilen zu können, ob der Winzer im Interesse der Genossenschaft handelt oder eigene Ziele verfolgt. Weingüter hingegen können nicht ohne weiteres auf Erfahrungswerte oder Informationen zurückgreifen wie es Genossenschaften möglich ist. Sie sind gezwungen, Informationen über ein persönliches Gespräch, Gespräche mit Dritten oder durch eine Besichtigung der Rebfläche zu sammeln. Dies verursacht höhere Transaktionskosten. Da für eine Genossenschaft meist nur Mitglieder für die Produktion von Premiumweinen zur Verfügung stehen, kann dies auch ein Nachteil sein. Das Interesse im Hinblick auf die Produktion von qualitativ hochwertigen Trauben kann nur durch hohe monetäre Anreize geweckt werden. Dabei kommt es durch Schaffung von Anreizen zu einer Erhöhung der Kosten. Hier wiederum liegt der Vorteil auf Seiten der Weingüter, die sich ihre Winzer selbst aussuchen und frei darüber entscheiden können, ob der Winzer in das gewünschte Profil passt oder nicht.

Die Begründung für die Selektion der Mitglieder oder der Vertragspartner lässt sich mit der PrinzipalAgent-Theorie erklären. Mithilfe der vorherigen Informationsbeschaffung über den Winzer soll die Gefahr der Wahl eines "falschen" Vertragspartners ("Adverse Selection") vermindert bzw. verhindert werden. Die Informationsbeschaffung reduziert die vorliegenden Informationsasymmetrien zwischen den Parteien. Somit können die Eigenschaften oder die angebotene Dienstleistung vom Prinzipal (Weingut / Genossenschaft) vor Vertragsabschluss besser bewertet werden. Gleichzeitig kann die "richtige" Wahl des Vertragspartners die Kontrollkosten sowie den Kontrollaufwand senken und Konflikte aufgrund unterschiedlicher Interessen vermeiden.

Aus Sicht der Transaktionskostentheorie lassen sich basierend auf den Ergebnissen der Untersuchung weitere Probleme erkennen. Bei nahezu der Hälfte der Weingüter und Genossenschaften werden keine genauen Vorgaben zur Bewirtschaftung der Rebfläche im Vertrag festgelegt. Diese werden in Form von vegetationsabhängigen Arbeitsanweisungen (schriftlich / mündlich) oder einer Vorgabe an die Zielmenge bzw. Qualität der Trauben vereinbart. Die genaue Ausarbeitung eines Vertrages, in welchem die Vorgaben an die Bewirtschaftung festgelegt sind, ist aufgrund des großen Einflusses und der Unsicherheit von externen Faktoren wie z. B. der Witterung viel zu aufwendig und nur schwer zu realisieren. Die Verträge sind deshalb "unvollkommen", weil der Vegetationsverlauf von Jahr zu Jahr unterschiedlich ist und die Transaktionskosten ex-post zu sehr steigen würden, wenn man sie ständig den sich ändernden Witterungseinflüssen anpassen müsste.

Die im Pacht- und Bewirtschaftungsvertrag oder Liefervertrag festgelegten weinbaulichen Maßnahmen sind aus Gründen der Umwelteinflüsse nicht immer eins zu eins umsetzbar. Problematisch ist hierbei die Unsicherheit der Transaktion. Deshalb sind die Vorgaben an die Bewirtschaftung der Rebfläche in gewisser Weise flexibel umzusetzen. Es besteht hierdurch die Möglichkeit auf externe Einflüsse zu reagieren, indem die "richtige" Wahl der qualitätsbeeinflussenden Maßnahme getroffen werden kann. Jedoch führen diese Flexibilität und Unsicherheit bei der vegetationsabhängigen Durchführung weinbaulicher Maßnahmen zu einer Erhöhung der Kontrollkosten oder es muss eine Kontrolle durch den Abnehmer der Trauben erfolgen, um beispielsweise die termingerechte Ausführung (Verhaltensunsicherheit) sicherzustellen. Die Ergebnisse der empirischen Untersuchung lassen darauf schließen, dass die Kontrolle der festgelegten weinbaulichen Maßnahmen bei allen Weingütern und Genossenschaften notwendig ist, weil die Gefahr von opportunistischem Verhalten seitens des Traubenproduzenten besteht und anderenfalls die Vorgaben nicht wie gewünscht umgesetzt werden.

Das Ausmaß und die Häufigkeit der Kontrolle haben aus Sicht der Theorie verschiedene Gründe. Dies kann anhand der Prinzipal-Agent-Theorie veranschaulicht werden. Das Weingut oder die Genossenschaft sind aufgrund der Informationsasymmetrien der Gefahr des "Moral Hazard" ausgesetzt. Diese tritt nach Vertragsabschluss auf. Es kann nicht ausgeschlossen werden, dass der Winzer die Vorgaben nicht wie vereinbart umsetzt und gewisse Spielräume ausnutzt ("Hidden action"). Die Kontrolle dient der Aufdeckung und der verbesserten Beobachtung von verborgenen Handlungen und Informationen des Agenten (Winzer). Anhand der 
Aussagen der Interviewpartner ist $\mathrm{zu}$ erkennen, dass sich einige Winzer opportunistisch verhalten oder nur den geringsten Aufwand betreiben, um die vorgegebenen Ziele zu erreichen ("Hidden Information"). Wie bereits erwähnt wurde, ist dieses Verhalten nur durch Kontrolle zu vermeiden und führt zu einer Erhöhung der Transaktionsbzw. Monitoring-Kosten.

Den Ergebnissen der Untersuchung zufolge, muss eine Kontrolle auch deshalb durchgeführt werden, weil zwischen dem Traubenproduzenten und Abnehmer unterschiedliche Interessen hinsichtlich der Notwendigkeit und Genauigkeit der Durchführung der Maßnahmen bestehen. Bei den Genossenschaften wird die Kontrolle bei knapp der Hälfte der Befragten durch einen Qualitätsmanager durchgeführt. Am Beispiel von Interviewpartner $\mathbf{M}$ (31:50 min) zeigt sich die Notwendigkeit der Kontrolle, um das Problem des "Moral Hazard" zu vermeiden. Mithilfe des Qualitätsmanagers kann die Herkunft unterschiedlicher Qualitätsergebnisse unter gleichen Anbaubedingungen identifiziert werden.

Die Kontrolle dient außerdem dazu, rechtzeitig eingreifen und die Qualität steuern zu können, wenn Vorgaben an die Bewirtschaftung nicht wie vereinbart umgesetzt werden oder Maßnahmen nicht ergriffen werden, um den externen Faktoren (z. B. Witterung) entgegenzuwirken. Durch regelmäßige Durchführung der Kontrollen soll der Winzer einen gewissen Druck verspüren, sodass dieser im Interesse des Abnehmers handelt. Dem Traubenproduzent soll sich darüber bewusst sein, dass er bei nicht ordnungsgemäßer Durchführung der Maßnahmen sanktioniert wird. Hier lässt sich der Einfluss von Macht durch Zwang erkennen. Der Betroffene muss bei Fehlverhalten mit einer Bestrafung rechnen. Durch Machteinfluss dieser Art soll das Verhalten des Winzers im Sinne des Unternehmens und deren Ziele beeinflusst bzw. gesteuert werden.

Die Ergebnisse der Untersuchung zeigen, dass die Häufigkeit der Kontrolle variiert. In langfristig bestehenden Kooperationen verfügen Weingüter und Genossenschaften über Erfahrungswerte und Informationen über ihre Winzer. Dadurch können sie besser beurteilen, wie oft eine Kontrolle der einzelnen Winzer notwendig ist. Der Kontrollaufwand kann hierdurch optimiert und somit reduziert werden. Die Ergebnisse der Weingüter zeigen, dass das Spektrum der Kontrolle von permanent durchgeführter Kontrolle bis hin zu gelegentlicher Kontrolle reichen. Das Ausmaß und die Häufigkeit der Kontrolle unterscheiden sich also in den einzelnen Betrieben. In diesem Zusammenhang spielt das Vertrauen gegenüber dem Traubenproduzenten eine wichtige Rolle. Der Kontrollaufwand und die Kontrollkosten sind bei Unternehmen, die ihren Winzern kein Vertrauen schenken, deutlich höher als bei denjenigen Unternehmen, die auf ein Vertrauensverhältnis setzen. Dies lässt sich beispielsweise an den Aussagen von Interviewpartner $\mathrm{C}$ verdeutlichen. Dieser vertraut seinen Winzern nicht und muss sie deshalb permanent kontrollieren. Die Untersuchung zeigt außerdem, dass die persönliche Beziehung zum Winzer großen Einfluss auf die Kosten und den Aufwand der Kontrolle hat. Das Leistungsvertrauen sowie das persönliches Vertrauen sind dabei wichtige Bestandteile der
Kooperation in einem Pacht- und Bewirtschaftungsvertrag zur Senkung der Kontrollkosten. Vertrauen ergibt sich durch langjährige und erfolgreiche Kooperation, sowie durch positive Erfahrungswerte mit dem Winzer und dem Erreichender vorgegebener Ziele (z. B. beim gelieferten Traubenmaterial). Die Häufigkeit der Transaktion wirkt sich positiv auf die Höhe der Transaktionskosten aus, da bestimmte Abläufe und Ziele bekannt sind.

Aus den Ergebnissen der Befragung lässt sich ableiten, dass die Anzahl der Winzer, die Größe des Unternehmens sowie die räumliche Entfernung zu den Rebflächen, Einfluss auf das Vertrauen und den Kontrollaufwand haben. Je geringer die Anzahl Winzer im Unternehmen, die Trauben über einen Pacht- und Bewirtschaftungs- oder Liefervertrag abliefern, desto vertrauenswürdiger ist auch die Beziehung zwischen Abnehmer und Traubenproduzent. Liegen zwischen der bewirtschafteten Rebfläche und dem betreffenden Unternehmen große räumliche Entfernungen, muss das Vertrauen gegenüber dem Winzer hoch sein, damit der Kontrollaufwand minimiert werden kann. Vertrauen sowie eine gute persönliche Beziehung sollen in diesem Fall auch die Gefahr des "Moral Hazard" und des opportunistischen Verhaltens reduzieren. Der Abnehmer der Trauben ist ansonsten nicht in der Lage, den Winzer "lückenlos" zu überwachen, was dieser zu seinen Gunsten ausnutzen könnte. Allgemein bleibt festzuhalten, dass die Kontrolle der Rebfläche / des Winzers aufgrund von Unsicherheiten und der Gefahr von "Moral Hazard" zwingend notwendig ist. Vertrauen alleine ist nicht ausreichend, um sich vor opportunistischem Verhalten und den Problemfeldern der Prinzipal-Agent-Theorie zu schützen. Die Ergebnisse machen außerdem ersichtlich, dass Vertrauen einen positiven Effekt auf das Beziehungsverhältnis hat und die Transaktionskosten reduziert.

Die Kontrolle des angelieferten Traubenmaterials erfolgt hauptsächlich über die optische Beurteilung des Traubenabnehmers. Bei der Anlieferung der Trauben kann ebenso die Gefahr des "Moral Hazard" aufgrund der Informationsasymmetrie zwischen beiden Parteien entstehen. Zudem besteht die Gefahr von opportunistischem Verhalten seitens des Traubenproduzenten. Ein Beispiel aus den Ergebnissen zeigt, dass der Winzer versucht, qualitativ minderwertige Trauben unter einer Deckschicht gesunder Trauben zu verbergen. Mithilfe dieser Täuschung möchte der Winzer seinen Gewinn maximieren, indem er die Kosten für die Sortierung der Trauben reduziert. An diesem Beispiel lässt sich das Problem von "Hidden action" für den Prinzipal (Weingut / Genossenschaft) erkennen. Dies wiederum zeigt, weshalb zur Beseitigung der Informationsasymmetrien eine Kontrolle des Traubenmaterials erforderlich ist. Eine optische Überprüfung des Traubenmaterials ist im Vergleich zu einer zusätzlichen Kontrolle durch mehrere Personen über einen Sortiertisch relativ kostengünstig. Interviewpartner $\mathrm{H}$ beispielsweise hat großes Vertrauen in den Winzern. Dennoch betreibt er durch die zusätzliche Kontrolle über den Sortiertisch einen hohen Kontroll- und Kostenaufwand, um hohe Qualität (bzw. ausschließlich gesunde Trauben) in der Weinproduktion zu haben. Dies verdeutlicht erneut, dass die Kontrolle der angelieferten, 
selbst bei vorherrschendem Vertrauen wichtig ist, um die Arbeit des Winzers beurteilen zu können sowie die gewünschte Qualität sicherzustellen.

Die Kontrolle ist besonders wichtig ist, wo kritische Phasen in der Vegetation bestehen und es bei einer Unterlassung der Kontrolle die Qualität der Trauben negativ beeinflussen kann. Insbesondere nach der Blüte bis kurz vor der Weinlese sind die entscheidenden Maßnahmen zu treffen, und es sollte eine Kontrolle der Vorgaben wie z. B. der Entblätterung, der Traubenteilung oder der Entfernung fauler Trauben durchgeführt werden. Die Kosten variieren je nach Art und Häufigkeit der Kontrolle. Bei einigen Weingütern werden weitere Parameter, wie z. B. das Mostgewicht kontrolliert, um die davon abhängige Qualität und den Auszahlungspreis bestimmen zu können.

Die Ergebnisse in Hinblick auf Anreize und Motivation zeigen, dass Geld bzw. der Auszahlungspreis, sowohl bei den Weingütern als auch bei den Genossenschaften der größte Anreizmechanismus für den Winzer ist. Um den Winzer im Sinne des Unternehmens steuern $\mathrm{zu}$ können, werden vorwiegend materielle (monetäre) Anreize geschaffen und genutzt. Hier lässt sich der Einsatz von "Macht der Belohnung" beobachten. Gleichzeitig wird aufgrund des Anreizes "Macht durch Zwang" genutzt. Dem Winzer wird verdeutlicht, dass er bei nicht korrektem Verhalten oder Umsetzung der weinbaulichen Vorgaben eine Sanktionierung (Abschlag bei der Bezahlung) zu erwarten hat. Die Art der Bezahlung (pauschaler Betrag oder nach Menge und Qualität) beeinflusst ebenso das Verhalten des Winzers. Die Schaffung von Anreizen wird genutzt, um die Risiken und Gefahren des "Moral Hazard" $\mathrm{zu}$ reduzieren und eine Interessenangleichung zwischen dem Winzer und dem Abnehmer zu schaffen. Bei einem Großteil der befragten Weingüter und Genossenschaften sind weiche Faktoren wie Lob und Anerkennung ebenfalls von Bedeutung und werden genutzt, um den Winzer $\mathrm{zu}$ steuern und $\mathrm{zu}$ motivieren. Insbesondere in Genossenschaften wirkt sich Lob und Anerkennung deutlich positiv auf die Motivation der Winzer aus, weil dies auf Mitgliederversammlungen geschieht und Stolz auf Seiten des Winzers hervorruft. Dies stärkt die Identifikation mit der Genossenschaft und kann die Transaktionskosten, ähnlich wie Vertrauen, reduzieren.

\section{Lösungsansätze und empfehlungen}

In den meisten Fällen entstehen Probleme aus den Informationsasymmetrien zwischen dem Winzer und dem Traubenabnehmer. Die Prinzipal-Agent-Theorie bietet Lösungsmöglichkeiten, um diese Informationsasymmetrien, welche ex ante und ex post entstehen können, zu beseitigen sowie Interessen anzugleichen. Die Auswahl des richtigen Vertragspartners ist von großer Bedeutung für den späteren Kontrollaufwand und die Entstehung von Konflikten. Die Weingüter und Genossenschaften sind, der Gefahr der "Adversen Selektion" bzw. "falschen" Wahl des Vertragspartners ausgesetzt. Um dieser Gefahr entgegenzuwirken, werden die Maßnahmen des Signaling,
Screening und Self-Selection vorgeschlagen (Picot / Dietl, 1993). Durch Signaling (Signalgebung) kann der Agent (Winzer) seine Eigenschaften und Fähigkeiten gegenüber dem Prinzipal (Weingut / Genossenschaft) signalisieren und zeigen, dass er in der Lage ist, die Vereinbarungen umzusetzen. Die Signalgebung kann bspw. in Form von Qualifikationsbescheinigungen (Zeugnisse, Meisterbrief, etc.), über einen guten Zustand der Rebfläche oder durch gute Ergebnisse der Traubenqualität in den vergangen Jahren erfolgen. Als Screening bezeichnet man den Aufwand des Prinzipals (z. B. Weingut), der versucht, durch die Beschaffung von Informationen einen besseren Überblick über die Eigenschaften und Fähigkeiten des Agenten zu erhalten (Picot / Dietl, 1993). Es ist daher wichtig, dass sich der Unternehmer Informationen über den Traubenproduzenten einholt. Hierdurch kann besser beurteilt werden, ob dieser die notwendigen Fähigkeiten besitzt, um die Vorgaben an die Bewirtschaftung der Rebfläche wie gewünscht umzusetzen. Desweiteren können Informationen über die Interessen und weinbaulichen Fähigkeiten in einem persönlichen Gespräch mit dem Winzer gesammelt werden. Der Kontrollaufwand kann hierdurch verringert werden, wenn beide Vertragsparteien die gleichen Interessen und Vorstellungen in Bezug auf den Weinanbau haben. Eine Befragung von ortskundigen Winzern oder eine Besichtigung der Rebfläche über deren Zustand wird vor Vertragsabschluss empfohlen. Bei SelfSelection werden dem Agent verschiedene Verträge zur Auswahl angeboten und diese sind vom Prinzipal so gestaltet, dass der Agent seine Qualitätseigenschaften durch die Wahl eines Vertrages offenbart (Picot et al., 2001). Der Agent wählt unbewusst auf Basis seiner Qualitätseigenschaften den für sich effizientesten Vertrag aus.

In der Regel sind Pacht- und Bewirtschaftungsverträge über mehrere Jahre angelegt. Dieses Vertragsverhältnis kann nur schwer wieder aufgelöst werden. Hierzu müssen Abfindungszahlungen getätigt werden, welche mit Kosten verbunden sind und womöglich über den Kosten der Informationsbeschaffung liegen. Dies soll die Notwendigkeit der Informationsbeschaffung vor Vertragsabschluss noch einmal verdeutlichen. Dennoch ist nicht auszuschließen, dass es nach Vertragsabschluss zu Konflikten und Meinungsverschiedenheiten kommt.

Zur Vermeidung von "Hidden action" und "Hidden information" ("Moral Hazard") gilt es bestimmte Informations- und Kontrollsysteme einzurichten. Aus Sicht der Weingüter und Genossenschaften müssen deshalb die Vorgaben an die Bewirtschaftung der Rebfläche kontrolliert werden. Dies erfolgt über die Besichtigung der Rebfläche. Es empfiehlt sich, diese Kontrolle ab dem Zeitpunkt der Blüte bis zur Ernte verstärkt durchzuführen, weil hier die entscheidenden Maßnahmen getroffen werden müssen, um die Qualitätsziele zu erreichen. Die Beschäftigung eines Qualitätsmanagers in der Genossenschaft ist eine sehr gezielte Maßnahme gegen das "Moral Hazard" Problem, um den hohen Kontrollaufwand aufgrund der Vielzahl von Winzern zu bewältigen. Gleichzeitig kann dieser durch seine beratende Funktion 
zu einer Steigerung der Traubenqualität beitragen. Die Installation eines Qualitätsmanagers ist aus den genannten Gründen empfehlenswert. $\mathrm{Zu}$ beachten ist, dass der Qualitätsmanager fachliche Qualifikation und Kompetenz aufweist, um Akzeptanz bei den Winzern erreichen zu können.

Eine staatliche Einrichtung führt stichprobenartige Kontrollen des Pflanzenschutzes sowie dessen Dokumentation durch und unterstützt den Traubenabnehmer bei der Überprüfung. Bei der Traubenannahme gilt es, bestimmte Parameter durch eine optische oder analytische Kontrolle zu beurteilen, um der Gefahr des "Moral Hazard" entgegenzuwirken. Dazu zählen bspw. Gesundheitszustand der Trauben, Färbung der Beerenhaut, physiologische Reife oder Mostgewicht. Zur Kontrolle bzw. Erfassung weiterer Parameter sind Analysegeräte ("Grapescan") notwendig, welche aufgrund des hohen Investitionsaufwands bei kleineren Unternehmen jedoch nicht in Relation zum Nutzen stehen.

Ist eine lückenlose Kontrolle und Überwachung des Vertragspartners aufgrund prohibitiv hoher Kosten nicht möglich, gilt es bestimmte Anreizmechanismen in Form von Belohnung oder Sanktionierung einzusetzen. Ziel dabei ist es, eine Interessenangleichung zu erreichen. Die Ergebnisse haben gezeigt, dass die Hauptmotivation der monetäre Anreiz ist. Das Auszahlungssystem kann jedoch auch ein Problem sein. Der Winzer neigt bei einem fixen Flächenbetrag dazu, die Vorgaben nur mit dem notwendigsten Aufwand umzusetzen. Bei einer Auszahlung nach Mostgewicht und Menge besteht die Gefahr, dass die gewünschte Qualität nicht erreicht wird. Der Winzer versucht in der Regel mehr Menge zu produzieren, worunter jedoch die Qualität leidet. Aus diesem Grund gilt es ein Anreizsystem zu schaffen, bei dem sich für beide Parteien eine Win-Win-Situation einstellt. Der Auszahlungspreis sollte in Relation mit der geleisteten Arbeit und der gelieferten Traubenqualität stehen. Es empfiehlt sich daher, eine Vorgabe an die abzuliefernde Menge und den Bereich des ZielMostgewichtes festzulegen. Sollte die Zielmenge oder der Bereich des Mostgewichtes nicht erreicht oder überschritten werden, ist dies in Form von Abschlägen bei der Auszahlung zu sanktionieren. Zu berücksichtigen ist, dass die Vorgaben aufgrund von Unsicherheiten oder Witterungseinflüssen nicht immer erfüllt werden können.

Zur Qualitätsbeeinflussung und Vermeidung von Konflikten und Problemen können auch soziale Aspekte genutzt werden. Die Kommunikation mit dem Winzer und die Zielvermittlung bzw. Einbindung in die Philosophie des Unternehmens fördern ebenfalls das Kooperationsverhältnis. Dies schafft eine Identifikation mit dem Unternehmen und wirkt opportunistischem Verhalten entgegen. Gemeinsamkeiten, wie z. B. Werte und Ziele können den Kostenaufwand für die AgencyKosten senken. Die persönliche Ebene und das Vertrauen sind ebenfalls wichtige Komponenten einer erfolgreichen Kooperation, welche aufgrund des häufigen Austausches über die Jahre hin wachsen. Bei Problemen und Konflikten empfiehlt sich der persönliche Kontakt mit dem Winzer. In einem Gespräch besteht für beide Parteien die Möglichkeit, sich auszutauschen und die Herkunft der
Probleme zu identifizieren. Dies wird nach Aussagen aller Interviewpartner der Weingüter und Genossenschaften durchgeführt. In einigen Genossenschaften wird ein Qualitätsmanager beschäftigt, der als Bindeglied zwischen den Winzern und der Geschäftsführung fungiert. Im Bereich des Konfliktmanagements ist der Qualitätsmanager mit einem Mediator zu vergleichen, welcher als neutrale Person zwischen den beiden Parteien schlichten soll (Proksch, 2010). Die Installation eines Qualitätsmanagers ist daher ein guter strategischer Lösungsansatz, um die Gefahren der Informationsasymmetrien durch die Kontrolle der Winzer zu vermeiden. Gleichzeitig kann dieser als Schlichtperson bei Konflikten zwischen dem Winzer und dem Vorstand vermitteln. Allerdings verursacht der Qualitätsmanager auch Kosten, die jedoch durch eine Qualitätssteigerung wieder amortisiert werden können.

\section{Zusammenfassung und ausblick}

Durch den Abschluss eines Pacht- und Bewirtschaftungsvertrages entsteht eine vertikale Kooperation zwischen dem Weingut und dem Traubenproduzenten. Aus Sicht der Genossenschaft als Unternehmen ist diese vertikale Kooperation ebenfalls durch den Liefervertrag für Premiumweine zu erkennen. Jedoch gibt es in einer solchen Beziehung auch Probleme und Konflikte. Das Ziel war es, diese Konflikte und Probleme durch Interviews mit Entscheidungsträgern der Weinwirtschaft zu erkennen und zu analysieren.

Die Ergebnisse zeigen, dass die meisten Probleme auf die Transaktionskostentheorie und auf die Prinzipal Agent Theorie zurückzuführen sind. Der Prinzipal (Weingut / Genossenschaft) ist hauptsächlich der Gefahr des "Moral Hazard" und der "Adversen Selektion" des Vertragspartners (Agent) ausgesetzt. Die Untersuchung hat gezeigt, dass eine Selektion der Mitglieder oder des Vertragspartners durchgeführt wird, um die falsche Wahl des Partners in einem Pacht- und Bewirtschaftungsvertrag oder Liefervertrag zu vermeiden. Um die vorliegenden Informationsasymmetrien $\mathrm{zu}$ beseitigen, fallen jedoch Transaktionskosten bzw. Agency-Kosten für die Kontrolle und Überwachung sowie für die Schaffung von Anreizen an. Die Kontrolle und Überwachung des Winzers ist sehr wichtig, um die Qualität der Trauben zu sichern. In diesem Zusammenhang kann Vertrauen in der Kooperation die Kosten für die Kontrolle und Überwachung deutlich reduzieren, jedoch eine Kontrolle nicht ersetzen. Hinsichtlich der Häufigkeit und dem Aufwand der Kontrolle waren große Unterschiede zu erkennen. Zur Steuerung des Verhaltens des Winzers werden Anreizsysteme in Form von monetären Anreizen (Geld, Garantien) oder in Form von Sanktionen (Zahlungsabschläge) genutzt. Zur Konfliktbewältigung wird das persönliche Gespräch mit dem Winzer gesucht und in Genossenschaften zusätzlich ein Qualitätsmanager installiert.

Es ist anzunehmen, dass Kooperationen in Form von Pacht- und Bewirtschaftungsverträgen aufgrund des starken Konzentrationsprozesses in der Weinwirtschaft zunehmen werden. Viele Weingüter benötigen mehr Fläche, um in Zukunft wirtschaftlich erfolgreich zu sein. Interessant wird es zu beobachten sein, wie sich eine mögliche Aufhebung des Anbaustopps in der 
Europäischen Union auf die Strukturen der Betriebe auswirken wird. Sollte es zu einer Aufhebung des Gesetzes kommen, besteht bei einer guten Wirtschaftskraft der Unternehmen die Möglichkeit, in Maschinen und Arbeitskräfte zu investieren sowie die Fläche nach Bedarf $\mathrm{zu}$ erhöhen und selbst zu bewirtschaften. Die Fixkosten, die durch die Anschaffung neuer Kapazitäten (z. B. Maschinen, Lagertanks, etc.) entstehen, könnten durch den nicht beschränkten Flächenzuwachs von Seiten des Gesetzgebers nun besser gedeckt werden. Voraussetzung ist jedoch, dass die Mehrproduktion an Wein auch auf dem Markt abgesetzt werden kann.

Abschließend ist zu sagen, dass eine Kooperation mit Winzern über Pacht- und Bewirtschaftungsverträge eine gute Möglichkeit darstellt, um den aus ökonomischer Sicht zukünftigen Herausforderungen der Betriebsvergrößerung entgegenzutreten.

\section{Literatur}

Coase, R.H., 1937: The Nature of the Firm, in: Economica, 4, S. 386-405

Demsetz, H., 1967: Toward a Theory of Property Rights. The American Economic Review, 57, No. 2, Papers and Proceedings of the Seventy-ninth Annual Meeting of the American Economic Association, 1967, S. 347-359

Engels, A., 2002: Genossenschaftliche Kooperationen im Weinbau: Ein Beitrag zur Vereinbarkeit genossenschafts- und unternehmenstheoretischer Erklärungen der Kooperation. Dissertation, Marburg 2002, S. 9-110

El-Ansary, A.L., Stern, L.W. (1972). Power measurement in the distribution channel, Journal of Marketing Research, 9 (1), S. 47-52

French, J.R.P., Raven, B., 1959: The Bases of Social Power. In: Studies of Social Power, edited by Cartwright, D., Ann Arbor, MI: University of Michigan Press, S. 150-167

Furubotin, E.G., Pejovich, S., 1972: Property Rights and Economic Theory: A Survey of Recent Literature. Journal of Economic Literature, 10, No. 4, 1972, S. 1137-1162

Hanf, J., Dautzenberg, K., 2008: Vertikale Kooperationen in der Agrar- und Ernährungswirtschaft Herausforderungen für das Management. In: Berichte über Landwirtschaft, Zeitschrift für Agrarpolitik und Landwirtschaft, Band 86 (1), 2008, S. 103-112

Hart, O., Moore, J., 1990: Property Rights and the Nature of the Firm. Journal of Political Economy, 98, Nr. 6, S. 1119-1158

Hendrikse, G., Bijman, J., 2002: Ownership Structure in Agrifood Chains: The Marketing Cooperative. American Journal of Agricultural Economics, 84, Nr.1, S. 104-119

Jensen, M., Meckling, W., 1976: Theory of the Firm: Managerial Behavior, Agency Costs and Ownership
Structure. Journal of Financial Economics, 3, No. 4, Seite 305-360

Kromrey, H., 2000: Empirische Sozialforschung. 9. Auflage, Opladen, S. 364

Kühlmann, T.M., 2008: Opportunismus, Vertrauen und Kontrolle in internationalen Geschäftsbeziehungen. In: E. Jammal (Hrsg.), Vertrauen im interkulturellen Kontext. Springer Verlag, S. 51-67

Kunkel, P.L., Peterson, J.A., Mitchell, J.A., 2009A: Agricultural Marketing Contracts. Farm Legal Series, University of Minnesota, Juni 2009, S.1-4

Kunkel, P.L., Peterson, J.A., Mitchell, J.A., 2009B: Agricultural Production Contracts. Farm Legal Series, University of Minnesota, Juni 2009, S.1-5

Mayring, P., 2002: Einführung in die qualitative Sozialforschung. 5. Auflage, Weinheim

Oberhofer, J., 2007: Mit Bewirtschaftungsverträgen in die Zukunft?. Bericht im Rahmen der 50. Rheingauer Weinbauwoche, 15. - 19. Januar 2007, S. 1-5

Picot, A., Dietl, H., 1993: Neue Institutionenökonomie und Recht. In: C. Ott/ H. Schäfer (Hrsg.): Ökonomische Analyse des Unternehmensrecht. Beiträge zum 3. Travemünder Symposium zur ökonomischen Analyse des Rechts. Physica Verlag Heidelberg, S. 306-330

Picot, A., Reichwald, R., Wigand, R.T., 2001: Die grenzenlose Unternehmung. Information, Organisation und Management. Lehrbuch zur Unternehmensführung im Informationszeitalter, 4. Auflage, Wiesbaden 2001

Proksch, S., 2010: Konfliktmanagement im Unternehmen, Mediation als Instrument für Konflikt- und Kooperationsmanagement am Arbeitsplatz. Heidelberg 2010, Springer Verlag, S. 31-58

Raven, B.H., Kruglanski, A.W. (1970): Control and power, in: Swingle, P. (eds): The Structure of Conflict, Academic, New York, NY, S.. 69-109

Schramm, M., Spiller, A., Staak, T., 2004: Brand Orientierung in der deutschen Ernährungsindustrie, Erfolgsdeterminanten der Markenführung am Beispiel genossenschaftlicher Hersteller. Deutscher Universitäts-Verlag, 2004, S. 52-72

Schreyögg, G., 2003: Prinzipal-Agenten-Beziehung in Organisationen. Wiesbaden : Gabler

Spiller, A., Schulze, B., 2006: Selbstbestimmte Landwirtschaft versus vertragsgebundener Rohstofflieferant: Einstellungen deutscher Landwirte zur Vertragsproduktion. In: Schriftenreihe "Landwirtschaft und Landschaftspflege in Thüringen", H. 9/2006, herausgegeben als Tagungsband anlässlich der "Wissenschaftlichen Tagung 2006", Jena, S. 44-60

Steinmetz, V., 2009: Bin ich's nun oder bin ich's nicht?. In: Fachzeitschrift "Badischer Winzer", Dezember 2009 , S. $25-26$

Williamson, O.E., 1990: Die ökonomischen Institutionen des Kapitalismus - Unternehmen, Märkte, Kooperationen. Mohr Siebeck Verlag, Tübingen 1990, S. 21-146 